\title{
Outcomes in pregnancies with a confined placental mosaicism and implications for prenatal screening using cell-free DNA
}

\author{
Francesca Romana Grati, MSc, PhD (1) ${ }^{1}$, Jose Ferreira, MD, PhD ${ }^{2,3,4}$, Peter Benn, DSc, PhD ${ }^{5}$, \\ Claudia Izzi, MD ${ }^{6}$, Federica Verdi, MD ${ }^{7}$, Elena Vercellotti, $\mathrm{MD}^{8}$, Cristina Dalpiaz, $\mathrm{MD}^{9}$, \\ Patrizia D'Ajello, $\mathrm{MD}^{8}$, Elisa Filippi, $\mathrm{MD}, \mathrm{PhD}^{10}$, Nicola Volpe, MD, PhD ${ }^{11}$, Francesca Malvestiti, $\mathrm{MSc}^{1}$, \\ Federico Maggi, MSc, $\mathrm{PhD}^{1}$, Giuseppe Simoni, MSc, $\mathrm{PhD}^{1}$, Tiziana Frusca, MD, PhD ${ }^{11}$, \\ Gaetana Cirelli, MD ${ }^{12}$, Gabriella Bracalente, $\mathrm{MD}^{10}$, Antonino Lo Re, MD ${ }^{9}$, Daniela Surico, $\mathrm{MD}^{8}$, \\ Tullio Ghi, MD, PhD ${ }^{11}$ and Federico Prefumo, MD, PhD ${ }^{6}$
}

Purpose: To assess the association between confined placental mosaicism (CPM) and adverse pregnancy outcome.

Methods: A retrospective cohort study was carried out evaluating the outcome of pregnancies with and without CPM involving a rare autosomal trisomy (RAT) or tetraploidy. Birthweight, gestational age at delivery, fetal growth restriction (FGR), Apgar score, neonatal intensive care admission, preterm delivery, and hypertensive disorders of pregnancy were considered.

Results: Overall 181 pregnancies with CPM and 757 controls were recruited. Outcome information was available for $69 \%$ of cases $(n=124)$ and $62 \%$ of controls $(n=468)$. CPM involving trisomy 16 (T16) was associated with increased incidence of birthweight $<3$ rd centile $(P=0.007$, odds ratio $[\mathrm{OR}]=11.2,95 \%$ confidence interval $[\mathrm{CI}]=2.7-47.1)$ and preterm delivery $(P=0.029, \mathrm{OR}=$ $10.2,95 \% \mathrm{CI}=1.9-54.7)$. For the other RATs, an association with prenatally diagnosed FGR was not supported by birthweight data and there were no other strong associations with adverse outcomes.

Conclusion: Excluding T16, the incidence of adverse pregnancy outcomes for pregnancies carrying a CPM is low. RATs can also be identified through genome-wide cell-free DNA screening. Because most of these will be attributable to CPMs, we conclude that this screening is of minimal benefit.

Genetics in Medicine (2020) 22:309-316; https://doi.org/10.1038/s41436019-0630-y

Keywords: rare autosomal trisomies; confined placental mosaicism; low birthweight; pregnancy complications; genome-wide cfDNA test

\section{INTRODUCTION}

Confined placental mosaicism (CPM) is defined as the presence of a chromosomal abnormality in the placenta but not in the fetus. ${ }^{1,2}$ This condition is detected when cytogenetic analysis of a chorionic villus sample (CVS) shows the presence of cell lines with normal and abnormal karyotypes but follow-up analysis on amniotic fluid cells or fetal tissue is normal. Depending on the distribution of the abnormal cell line in the two placental layers, three different types of CPM can be identified: type I, with the abnormal cell line identified in the cytotrophoblast only; type II, with the abnormality in

the mesenchyme only; and type III, with both placental layers containing the abnormal cell line.,

The most common type of CPM involves a trisomy/disomy. The mechanisms generating such fetoplacental discrepancies are generally either a mitotic chromosome segregation error occurring in an initially chromosomally normal conceptus, or a meiotic error resulting in trisomy with subsequent postzygotic "trisomic rescue." Meiotic origin is generally associated with high levels of trisomy in trophoblasts. A model for this association is mosaic trisomy 16 (T16) that is usually detected as CPM type III and is associated with fetal

\footnotetext{
${ }^{1}$ Unit of Research and Development, Cytogenetics and Medical Genetics TOMA, Advanced Biomedical Assays, Impact Lab Group, Busto Arsizio, Varese, Italy; ${ }^{2}$ Faculty of Medicine, Eduardo Mondlane University, Maputo, Mozambique; ${ }^{3}$ Department of Obstetrics and Gynecology, Maputo Central Hospital, Maputo, Mozambique; ${ }^{4}$ Genomed S.A., Warsaw, Poland; ${ }^{5}$ Department of Genetics and Genome Sciences, University of Connecticut Health Center, Farmington, CT, USA; ${ }^{6}$ UO Diagnosi Prenatale, Azienda Ospedaliera Spedali Civili, Brescia, Italy; ${ }^{7}$ Ostetricia e Ginecologia, Ospedale di Bolzano, Bolzano, Italy; ${ }^{8}$ Università del Piemonte Orientale, Novara, Italy; ${ }^{9}$ UO Ostetricia e Ginecologia, Clinica Pederzoli, Peschiera del Garda, Italy; ${ }^{10}$ UOC Ginecologia e Ostetricia, Osp. Cà Foncello Treviso, Treviso, Italy; ${ }^{11}$ Department of Medicine and Surgery, Unit of Surgical Sciences, Obstetrics and Gynecology, University of Parma, Parma, Italy; ${ }^{12}$ Ostetricia e Ginecologia, Ospedale Santorso AULSS7 Pedemontana (VI), Santorso, Italy. Correspondence: Francesca Romana Grati (fgrati@tomalab.com)
} 
abnormality, fetal growth restriction (FGR), and other pregnancy complications. ${ }^{5}$ Meiotic CPM may also be associated with the presence of fetal uniparental disomy (UPD), which is defined as a pair of homologous chromosomes derived from only one parent. UPD is of particular clinical interest when it involves chromosomes $6,7,11,14,15$, or 20 because these chromosomes carry imprinted regions that are associated with defined syndromes. Phenotypes can include fetal growth restriction (FGR) and reduced postnatal growth. ${ }^{6}$

The prevalence of mosaicism in CVS for viable pregnancies at $10-12$ weeks of gestation is approximately $2 \% .^{3,7-9}$ When a rare autosomal trisomy (RAT, defined as any autosomal trisomy other than T21, T18, and T13) is detected in CVS, in 97\% of the cases it is a CPM. ${ }^{3}$ Although this is a common finding, the possible association between CPM and adverse pregnancy outcome is unclear and counseling is therefore challenging. Some authors reported a higher risk of adverse pregnancy outcome, mainly FGR, ${ }^{10-15}$ while others did not. ${ }^{16-22}$ Many studies had a small number of cases, did not consistently combine the analyses of the direct preparation with the longterm cultures, or lacked a confirmatory amniocentesis to discriminate between CPM and true fetal mosaicism (TFM) in all cases.

Because of the limitations of past studies and the controversy around the significance of CPM, we undertook a retrospective cohort study based on review of pregnancy outcomes in affected pregnancies and in controls to assess the association between CPM and adverse pregnancy outcome.

\section{MATERIALS AND METHODS}

This retrospective cohort study was based on medical record review of women who underwent CVS between May 2000 and January 2018 at seven Italian referral centers for prenatal diagnosis. The villus samples of each patient were submitted to a single laboratory, which conducted the cytogenetic analysis by means of both cytotrophoblast and mesenchymal karyotyping. $^{23,24}$ Informed consent for the analyses was obtained from all subjects. The most common indications for CVS in the entire data set were maternal anxiety/elective decision (women <35 years) or advanced maternal age (approximately $80 \%$ of the cases). All cases of RATs or with tetraploidy greater than $20 \%$ observed during the study period were included. A mosaicism was defined as the presence of a mosaic abnormality (MA) or nonmosaic abnormality (NMA) in one of the two placental tissues, or MA in both tissues or NMA in one tissue and MA in the other. Rare cases with both abnormal nonmosaic layers were also included when associated with normal ultrasound findings. TFM was defined as the presence of at least two colonies from two AF cultures showing the same abnormality as that previously observed at CVS.

All patients with a diagnosis of mosaic of CVS involving a RAT or a tetraploidy had a genetic consultation and were offered a follow-up amniocentesis with UPD analysis (for cases with trisomy $6,7,11,14,15,20)$ and a detailed ultrasound anomaly scan. Methods for cytogenetic and UPD analyses have been described in detail in elsewhere ${ }^{3}$ (Supplementary Materials and Methods).

Inclusion criteria were (1) singleton pregnancy; (2) absence of ultrasound abnormalities (except increased nuchal translucency or soft markers); (3) an abnormal cell line showing a RAT, tetraploidy, or a whole autosomal arm imbalance detected in the two placental layers (type III), or in the longterm culture (type II) or in direct preparation only (type I); (4) a follow-up amniocentesis that showed a normal karyotype. Exclusion criteria were (1) twin pregnancy, (2) confirmed TFM on amniocytes, (3) incomplete CVS analysis (direct or long-term culture, only), (4) CPM for common trisomies $(\mathrm{T} 21,18,13)$ or sex chromosome aneuploidies.

Control subjects from singleton pregnancies with no evidence of CPM were selected from the laboratory database by identifying four contemporaneous cases (two male and two female fetuses) with similar maternal age, gestational age at the time of the procedure, and indication for prenatal diagnosis. Cases and controls were coded and blinded prior to medical record review. Each clinical center had an assigned analyst for the extraction of follow-up data from the medical records.

The following pregnancy outcome information was considered:

1. Birthweight percentile and birthweight below the 3rd percentile (frequently used as a classifier for small for gestational age).

2. Prenatal diagnosis of FGR, based on medical chart documentation.

3. Apgar score at 5 minutes.

4. Admission to a neonatal intensive care unit (NICU), excluding the subjects with prenatal assignment of FGR (outcome 2).

5. Hypertensive disorders (HD) of pregnancy (HELLP syndrome, preeclampsia, pregnancy induced hypertension).

6. Spontaneous preterm delivery (PTD) including cases with or without prior premature rupture of membranes.

7. Fetal anomaly, stillbirth, miscarriage, or termination of pregnancy.

8. Other, less common, pregnancy complications (e.g., acute chorioamnionitis, cholestasis, gestational diabetes, abruptio placentae, placenta previa).

We used INTERGROWTH-2 $1^{\text {st }}$ project charts to calculate birthweight percentile from gestational age at birth, birthweight, and newborn sex, because they are independent of ethnicity, or any other maternal factors that have been previously suggested to influence fetal growth. ${ }^{25}$

Institutional review board (IRB) approval was obtained from TOMA laboratory IRB (\#0000023/2018).

\section{Statistical data analysis}

The associations between the diagnosis of CPM and each outcome variable were assessed by an independent analyst, after de-coding and classification into cases and controls. Mann-Whitney exact test was used for numeric and ordinal 
outcome variables and exact Chi-square test for categorical binary outcome variables. Linear regression was used for associations between the degree of abnormal cell lines in placenta and quantitative outcome variables.

Associations between CPM and outcomes were assessed for CPMs involving a RAT (excluding T16) or a whole autosomal arm imbalance, for CPMs with tetraploidy only, and CPM with T16 only.

Birthweights (outcome 1 above) were treated as quantitative (interval) variables and categorical binary variables, respectively. Apgar scores (outcome 3) were treated as ordinal variables; all the other outcomes were treated as categorical binary variables.

Statistical testing was carried out using PASW Statistics (version 18, 30 July 2019, New York, NY, USA). A $P$ value $<0.05$ was considered significant. Correction for multiple testing was not applied (see Supplementary Materials and Methods).

\section{RESULTS}

In this series of 76,104 CVS, 1603 cases (2.1\%) displayed a chromosomal mosaicism. Of these, 1212 cases were subsequently investigated by a midtrimester amniocentesis. In 1051 of them $(86.7 \%)$ the amniotic fluid karyotype showed no evidence of chromosome abnormality; they were therefore classified as CPMs. Of these, 443 cases (42.2\%) involved a RAT (176 CPM type I, 200 CPM type II, and 54 CPM type III), 72 cases $(5.9 \%)$ involved a tetraploidy (32 CPM type I, 15 CPM type II, and 25 CPM type III), and 23 (1.9\%) a whole arm autosomal imbalance, mainly isochromosome (6 CPM type I, 10 CPM type II, and 3 CPM type III).

The seven participating centers accounted for 181 cases or $41 \%$ of the total CPM $(n=443)$ in the full data set. The remaining 262 cases were from multiple smaller referral locations where it was impractical to gather follow-up. There were 757 matched controls. One or more of the clinical outcomes were obtained for 124/181 (68.5\%) CPM cases and $468 / 757$ (62\%) controls. The missing outcomes were due to outpatient document disposal according to Italian regulations (after 10 years), or because the patient delivered in a different institution. Figure 1 summarizes the study design. There was no difference in maternal age distribution and in indications for testing between cases and controls (Table S1 and S2).

Figure 2 shows the number of the specific chromosome abnormalities present as CPM in the cases. We included the three cases with isochromosomes of $11 \mathrm{q}, 7 \mathrm{p}$, and $20 \mathrm{p}$ as RATs. Trisomies 2, 3, 7, and 16 were the most frequently encountered CPMs.

Table 1 summarizes the classification of the 124 CPMs (types I, II, and III) and the average percentage of the abnormal cell line in the affected placental layers. The relative proportions of the three CPM types in the study were similar to those seen in the full set of CVS cases. Most CPMs were types I and II. RATs that were present in both placental layers (type III) generally showed a higher percentage of abnormal cells. This agrees with the expectation that type III abnormalities are mostly of meiotic origin. ${ }^{5}$

UPD was investigated for CPMs involving imprinted chromosomes $(7,11,14,15$, or 20) (Table 2 ). The only UPD identified in this cohort was in one case of T14. That pregnancy was terminated due to the UPD and abnormalities identified by ultrasound.

Table 3 and S3 summarize the outcomes for the various types of CPM. For CPMs involving RATs (excluding trisomy 16), a statistically significant association was found with FGR (odds ratio [OR] 3.4; 95\% confidence interval [CI] 1.3-9.3). There was also a weak association with NICU admission excluding FGR (OR 3.3; 95\% CI 1.0-10.2) (Table S3). For NICU admission, the proportion of abnormal cells in cytotrophoblasts and mesenchyme was not statistically significant. There was no association with HD, PTD, or birthweight percentile (below third or when all birthweights were considered).

Because of literature suggestive of poor outcomes with CPM involving T16, this subgroup (11 cases) was analyzed separately. There was a statistically significant excess of cases with birthweight $<3$ rd percentile (OR 11.2; 95\% CI 2.7-47.1). However, when all birthweights were considered as quantitative outcomes, a significant correlation could not be demonstrated. CPM T16 cases also appeared to show an association with FGR (OR 8.4; 95\% CI 1.6-43.2). In addition, there was a statistically significant association with spontaneous PTD (OR 10.2; 95\% CI 1.9-54.7) and low Apgar score $(P=0.006)$.

CPMs involving T16 only were also analyzed to determine whether the proportion of abnormal cells in cytotrophoblast and mesenchyme was associated with birthweight. There were three T16 CPM cases that had a birthweight less than the 3rd percentile and all had a mosaicism level of $100 \%$ in both cytotrophoblasts and mesenchyme. There was only one other T16 case with this level of mosaicism that had a birthweight percentile $>3$ rd (24th percentile). The average percentage of T16 cells for the cases with a birthweight percentile $>3 \mathrm{rd}$ was $46 \%(\min 0 \%, \max 100 \%)$ in the mesenchyme and $40 \%$ (min $0 \%$, max $100 \%$ ) in the cytotrophoblast. There were three T16 cases whose birthweight percentile was above $90 \%$ and the levels of abnormal cells in cytotrophoblast and mesenchyme were $76 \%$ and $29 \%, 0 \%$ and $9 \%$, and $0 \%$ and $10 \%$, respectively. A linear regression analysis of abnormal cell percentage for all T16 cases with birthweight percentile showed an association in both mesenchyme (adjusted $r^{2}=$ 0.044; $P=0.03$ ) and cytotrophoblast (adjusted $r^{2}=0.07 P=$ 0.008 ) when analyzed independently. When analyzed in a multiple variable regression model, the $P$ value was 0.028 and the adjusted $r^{2}$ was 0.044 . These data suggest that the increased degree of trisomy in mesenchyme and cytotrophoblast explains only $4.4 \%$ of the variation in birthweight percentile and that the degree of mosaicism in cytotrophoblast has a bigger effect than the degree of mosaicism in mesenchyme. 


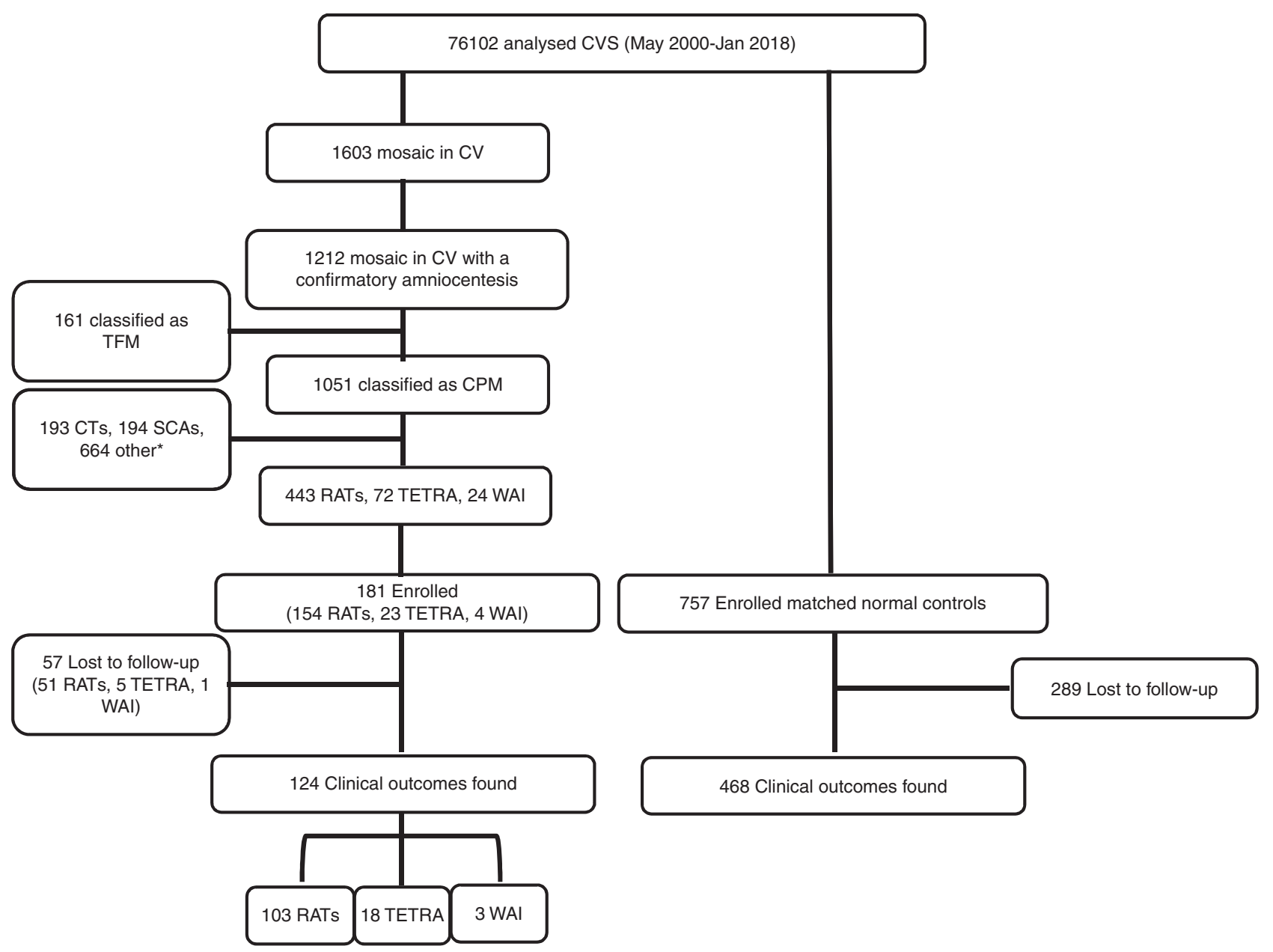

Fig. 1 Study design. CPM confined placental mosaicism, CTs common trisomies, CV chorionic villus, RATs rare autosomal trisomies, SCAs sex chromosome aneuploidies, TETRA tetraploidy, TFM true fetal mosaicism, WAI whole autosomal arm imbalance, *whole arm Xp or Xq trisomies, unbalanced structural rearrangements, monosomies, triploids.

There were only three miscarriages (all controls) and two stillbirths (one T16 caused by tearing of the umbilical cord, and one control). There were six elective pregnancy terminations, two of them in CPM cases (one in a CPM for T22 with $100 \%$ abnormal cells in both placental layers, and the other in a CPM for T14, in which talipes and UPD were present), and four in controls (one for increased nuchal translucency and subsequent detection of a cardiac abnormality, one with a cardiac anomaly, one with a dup 22q11, and one because of very early onset FGR). No other fetal abnormalities were recorded. These numbers of fetal anomalies, miscarriage, stillbirth, and terminations of pregnancy were insufficient for a meaningful statistical analysis. Similarly, there appeared to be only a small number of other pregnancy complications (data not shown) with insufficient numbers for formal statistical comparisons.

The data set included 18 cases with tetraploidy CPM. There was no evidence for a statistically significant association between this finding and any of the adverse outcomes analyzed.

\section{DISCUSSION}

In this study we have evaluated the clinical significance of CPM involving aneuploidies other than the common ones in CVS. Only T16 was found to be strongly associated with a risk for an adverse outcome $(\mathrm{OR}>8)$. CPMs involving RATs appeared to be significantly associated with FGR. However, except for those involving T16, there was no association with low birthweight less than the 3rd percentile or when considering all birthweight percentiles, suggesting that the FGR association for RATs (excluding T16) may have been spurious. For example, in this group, there were 21 pregnancies assigned as having FGR, of which only 13 had a birthweight $<10$ th percentile. Conversely, of 19 newborns with a birthweight percentile $<3 r$ d, only 8 were assigned as FGR. Heterogeneous criteria were used for defining FGR with differences between centers; some estimated fetal weight percentiles were calculated using Hadlock's charts ${ }^{26}$ whereas others used Yudkin's curves. ${ }^{27}$ Actual birthweight centiles are based on appropriate population standards and should constitute a more reliable measure of fetal growth. ${ }^{25}$ It is also 


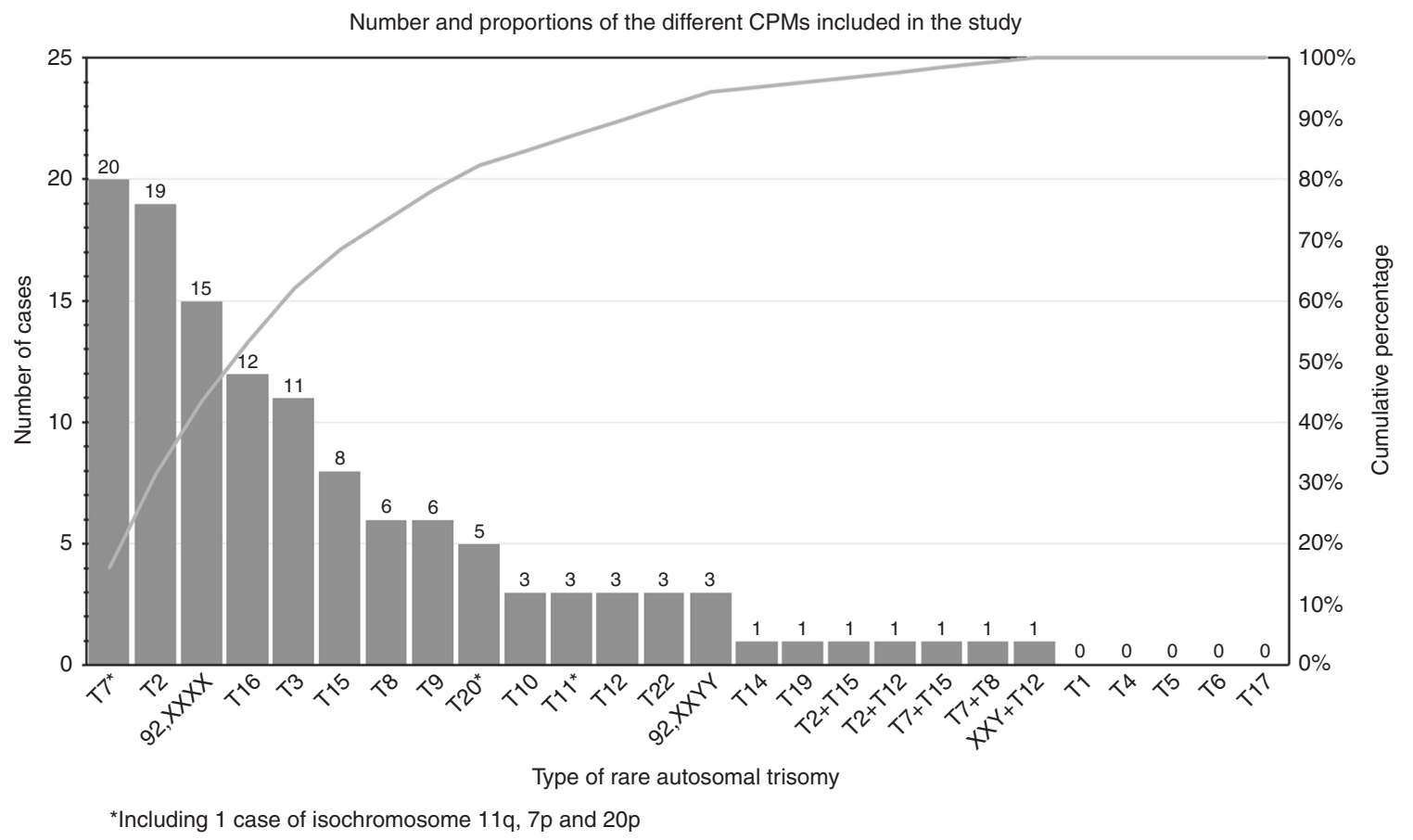

Fig. 2 Rare autosomal trisomies and tetraploid abnormalities included in the study. Arranged with most common abnormalities listed first. The red line denotes the cumulative number (\%). CPM confined placental mosaicism.

possible that the reporting $\mathrm{CPM}$ itself resulted in an ascertainment bias in the assignment of FGR, attributable to prior literature suggesting an association. The diagnosis of FGR, with possible bias, could also have been the indication for admission to the NICU. Therefore, we excluded subjects assigned as having FGR when considering the association between RATs and NICU admission. After this exclusion, NICU admission was found to be of borderline significance $(P=0.049)$ and this weak association could still be attributable to biased referral of cases to NICUs.

There is a substantial body of evidence to believe that CPM involving trisomy 16 is associated with FGR, prematurity, preeclampsia, and fetal abnormalities. ${ }^{28-31}$ In this study, which only included 12 cases, a significant association was found between CPM T16 and birthweight $<3$ rd percentile. Interestingly, the association between CPM T16 and the quantitative variable birthweight percentile failed to reach statistical significance. It is possible that CPM T16 causes a severe growth restriction in a small number of cases but in the full set of cases CPM has only a minor effect. Consistent with this, we observed that cases with birthweight percentile $<3 \mathrm{rd}$ were atypical in having $100 \%$ abnormal cells in both placental layers. No association was found with $\mathrm{HD}$ although preeclampsia is a known complication of these pregnancies. ${ }^{28,29}$ An association with PTD and low Apgar score was also identified for T16 CPM.

No association was found between CPM involving tetraploidy and any adverse outcome. Tetraploidies are commonly detected in cytotrophoblasts and counseling is challenging because this result might be an in vitro cultural artifact or a true clonal abnormality. ${ }^{3}$ This study shows that, in absence of fetal ultrasound abnormalities, these women can be reassured that pregnancy outcomes are like those in a control population.

Previous studies assessing the significance of CPM have been controversial with conflicting results, possibly due to differences in study design. The study methodology may be, at least in part, a contributing factor; there have been case-control studies that assessed whether CPM is more frequent in FGR pregnancies. ${ }^{11-13,20}$ Other studies assessed differences in clinical presentation, perinatal outcome, and postnatal growth and development between infants for FGR pregnancies with or without CPM. ${ }^{32}$ Finally, there have been cohort studies that assessed whether there is an increased risk of FGR and pregnancy complications in CPM compared with non-CPM matched pregnancies. ${ }^{16,17,21-23}$ The present study is of this latter type. Differences in sample size, with smaller samples being more prone to biases, may also explain the differences in conclusions.

Overall, the results of this study are reassuring: only CPM for T16 showed an increased risk for birthweight percentile $<3^{\text {rd }}$ and spontaneous PTD while for most CPMs for rare autosomal trisomies (excluding T16) detected prenatally by CVS analysis, the general health of the mother and fetus can be expected to be close to that seen for pregnancies with a normal CV karyotype. These results agree with those of Amor and collaborators ${ }^{21}$ who found no increase in prenatal complications or adverse neonatal outcomes.

\section{Strengths of the study}

This represents the most extensive cohort to date for CPM pregnancy outcomes. All cytogenetic analyses were carried 


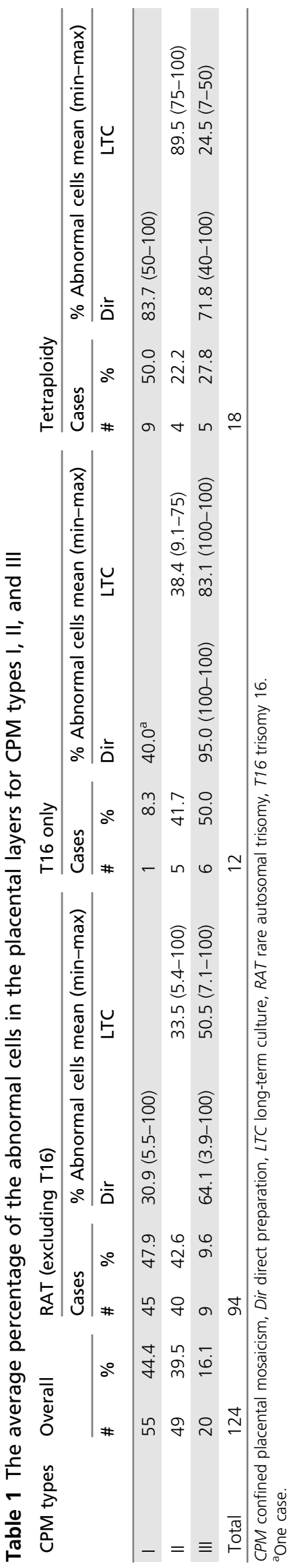

Table 2 Evaluation for uniparental disomy (UPD) in cases with CPM for trisomy or partial trisomy for chromosomes 6, $7,11,14,15$, or 20

\begin{tabular}{llll}
$\begin{array}{l}\text { Imprinted } \\
\text { chromosome }\end{array}$ & $\begin{array}{l}\text { Number } \\
\text { present }\end{array}$ & $\begin{array}{l}\text { Tested cases } \\
(n)\end{array}$ & $\begin{array}{l}\text { UPD detected } \\
(n)\end{array}$ \\
\hline Trisomy 6 & 0 & 0 & 0 \\
\hline Trisomy 7 & 21 & 15 & 0 \\
Trisomy 11 & 3 & 3 & 0 \\
\hline Trisomy 14 & 1 & 1 & 1 \\
\hline Trisomy 15 & 10 & 10 & 0 \\
\hline Trisomy 20 & 5 & 3 & 0 \\
\hline Total & 39 & 32 & 1 \\
\hline
\end{tabular}

CPM confined placental mosaicism.

out at a single laboratory with uniform policies for systematic direct preparation, long-term culture, and follow-up amniocenteses. The study included a large number of matched controls $(n=468)$. Follow-up was gathered in a blinded fashion with a high completion rate (63\%). Most patients did not receive prior ultrasound or maternal serum screening that could preselect cases with particular types of CPM.

\section{Limitations of the study}

Due to the retrospective nature of this study, there were no standard diagnostic criteria for FGR during the study period. Furthermore, except for CPM involving trisomy 16 and tetraploidies, we could not investigate associations for each individual chromosome abnormality involved due to insufficient power. Therefore, additional studies are needed to determine if T16 CPM is the only specific CPM that is associated with growth restriction. We cannot exclude that the possibility that cases with follow-up were biased toward those with either abnormal or normal pregnancy outcomes. For example, pregnancies terminated following the ultrasound detection of abnormality prior to confirmatory amniocentesis would not be included. Finally, as shown in Supplementary Table S3, our sample size was only sufficient to identify strong associations between the CPM RATs and the assessed outcomes. We could not exclude small associations, such as OR less than 3 to 10 .

\section{Implications for cfDNA screening}

The results of the present study have implications on the clinical utility of RAT detection through genome-wide cellfree DNA (cfDNA) testing. Direct CVS analysis and cfDNA are both based on the trophoblast cell lineage and both will identify RATs, most of which will be CPMs. ${ }^{33}$ The main advantage claimed for detecting RATs through cfDNA is because of the association with pregnancy complications. ${ }^{34-36}$ RATs can be readily detected by genome-wide technologies in which counting data are already available. Therefore, the relevant question is whether there is additional useful information that could be extracted by looking for RATs when the cfDNA test is being done anyway. 
Table 3 Summary of all association studies between CPMs and outcomes

\begin{tabular}{|c|c|c|c|c|c|}
\hline Outcome & $\begin{array}{l}\text { CPM (RATs } \\
\text { excluding T16) } \\
N=94\end{array}$ & $\begin{array}{l}\text { CPM (tetraploidy } \\
\text { only) } N=18\end{array}$ & $\begin{array}{l}\text { CPM (T16 } \\
\text { only) } N=12\end{array}$ & Controls $N=468$ & $\begin{array}{l}\text { Significant } \\
\text { associations }\end{array}$ \\
\hline Binary outcome variables $\left(X^{2}\right)$ & \multicolumn{3}{|c|}{ \# Abnormal outcome/total (\%) } & $\begin{array}{l}\text { \# Abnormal outcome/ } \\
\text { total }(\%)\end{array}$ & \\
\hline \multirow[t]{2}{*}{ Birthweight percentile $<3 r d$} & $3 / 87(3.4 \%)$ & $0 / 17$ & $3 / 11(27.3 \%)$ & $13 / 401(3.2 \%)$ & T16 only \\
\hline & $P 1.0$ & $P 0.672$ & $\mathrm{P} \underline{0.007}$ & & \\
\hline FGR & $\mathrm{P} \underline{0.018}$ & $P 0.072$ & $\mathrm{P} \underline{0.039}$ & & t16) T16 only \\
\hline \multirow[t]{2}{*}{ NICU admission (excluding FGR) } & $5 / 84(6 \%)$ & $1 / 18(5.6 \%)$ & $1 / 10(10 \%)$ & $8 / 420(1.9 \%)$ & RATs \\
\hline & P $\underline{0.049}$ & $P 0.317$ & $P 0.192$ & & (excluding T16) \\
\hline \multirow[t]{2}{*}{ HD } & $6 / 92(6.5 \%)$ & $1 / 18(5.6 \%)$ & $1 / 12(8.3 \%)$ & $20 / 428(4.7 \%)$ & None \\
\hline & $P 0.60$ & $P 1.0$ & P 1.0 & & \\
\hline \multirow[t]{2}{*}{ Birthweight percentile } & $\begin{array}{l}N=87(49 \\
52,26-78)\end{array}$ & $\begin{array}{l}N=17(44 \\
37,25-63)\end{array}$ & $\begin{array}{l}N=11(35 \\
89,2-91)\end{array}$ & $N=401(55,50,31-81)$ & None \\
\hline & $P 0.257$ & $P 0.186$ & $P 0.181$ & & \\
\hline \multirow[t]{2}{*}{ Apgar score at 5 minutes } & $\begin{array}{l}N=87(10 \\
1,9-10)\end{array}$ & $N=17(9,1,9-10)$ & $\begin{array}{l}N=11(9 \\
0,9-9)\end{array}$ & $N=408(10,1,9-10)$ & T16 only \\
\hline & $P 0.477$ & $P 0.120$ & P 0.006 & & \\
\hline
\end{tabular}

${ }^{a} P$ values compare the CPM group versus controls.

$C P M$ confined placental mosaicism, FGR fetal growth restriction, HD hypertensive disorders of pregnancy, IQR interquartile range, NICU neonatal intensive care unit, $P T D$ preterm birth, T16 trisomy 16.

Statistically significant $p$-values are in bold underlined $p<0.05$

Initial studies with genome-wide cfDNA screening have reported high rates of FGR but these need to be interpreted cautiously because they may be subject to ascertainment biases similar to those we encountered in this study. ${ }^{37,38}$ Our data indicate that $0.41 \%$ of cases show a CPM (type I or III) involving a RAT in cytotrophoblasts and, of these, ${ }^{3} 6 / 98$ (6.1\%) will be associated with a birthweight percentile $<3 \mathrm{rd}$ meaning that routine detection of CPM in a general population might result in identification of $0.41 \% \times 6.1 \%=$ $0.025 \%$ of cases with birthweight percentile $<3 \mathrm{rd}$. This represents only $0.025 \% / 3 \%=0.8 \%$ of all such cases present in the population. Under the assumption that CVS and cfDNA have comparable efficacy, the yield of low birthweight babies identifiable through a RAT would therefore seem to be minimal. We therefore conclude that cfDNA screening is unlikely to be an effective screening test for low birthweight.

This study shows that T16 is the only individual RAT that may be worth looking for and reporting through cfDNA screening. However, even for mosaic T16, pregnancy outcomes are highly variable and optimal prenatal management has not been defined. Genome-wide cfDNA screening will also potentially lead to the identification of some cases with UPD, but this yield is expected to be very low. ${ }^{3}$ Cell-free DNA screening will also identify nonmosaic RATs, which are associated with early fetal losses, but these are neither preventable nor actionable. Screening for RATs is not endorsed by the American College of Medical Genetics and Genomics. ${ }^{39}$

\section{SUPPLEMENTARY INFORMATION}

The online version of this article (https://doi.org/10.1038/s41436019-0630-y) contains supplementary material, which is available to authorized users.

\section{DISCLOSURE}

P.B. is a consultant for and holds stock options in Natera, Inc. F.R. G. and F. Malvestiti are full-time employees of TOMA laboratory, Impact Lab Group without ownership shares. F.R.G. is an expert panel member for Roche and consultant for Menarini Biomarkers. G.S. is a consultant for TOMA laboratory, Impact Lab Group. F. Maggi holds stock options in TOMA laboratory, Impact Lab Group. F.P. received consulting fees from Temas srl for an economic evaluation study of cell-free fetal DNA screening in the Italian national health service. The other authors declare no conflicts of interest.

Publisher's note: Springer Nature remains neutral with regard to jurisdictional claims in published maps and institutional affiliations.

\section{REFERENCES}

1. Kalousek DK, Dill FJ. Chromosomal mosaicism confined to the placenta in human conceptions. Science. 1983;221:665-667.

2. Simoni G, Sirchia SM. Confined placental mosaicism. Prenat Diagn. 1994;14:1185-1189.

3. Malvestiti F, Agrati C, Grimi B, et al. Interpreting mosaicism in chorionic villi: results of a monocentric series of 1001 mosaics in chorionic villi with follow-up amniocentesis. Prenat Diagn. 2015;35:1117-1127. 
4. Grati FR, Malvestiti F, Branca L, Agrati C, Maggi F, Simoni G. Chromosomal mosaicism in the fetoplacental unit. Best Pract Res Clin Obstet Gynaecol. 2017;42:39-52.

5. Robinson WP, Barrett IJ, Bernard L, et al. Meiotic origin of trisomy in confined placental mosaicism is correlated with presence of fetal uniparental disomy, high levels of trisomy in trophoblast, and increased risk of fetal intrauterine growth restriction. Am J Hum Genet. 1997;60:917-927.

6. Miozzo M, Simoni $G$. The role of imprinted genes in fetal growth. Biol Neonate. 2002;81:217-228.

7. Ledbetter DH, Zachary JM, Simpson JL, et al. Cytogenetic results from the U.S. collaborative study on CVS. Prenat Diagn. 1992;12:317-345.

8. Pittalis MC, Dalpra L, Torricelli F, et al. The predictive value of cytogenetic diagnosis after CVS based on 4860 cases with both direct and culture methods. Prenat Diagn. 1994;14:267-278.

9. Hahnemann JM, Vejerslev LO. European collaborative research on mosaicism in CVS (EUCROMIC)-fetal and extrafetal cell lineages in 192 gestations with CVS mosaicism involving single autosomal trisomy. Am J Med Genet. 1997;70:179-187.

10. Roland B, Lynch L, Berkowitz G, Zinberg R. Confined placental mosaicism in CVS and pregnancy outcome. Prenat Diagn. 1994;14:589-593.

11. Wilkins-Haug L, Roberts DJ, Morton CC. Confined placental mosaicism and intrauterine growth retardation: a case-control analysis of placentas at delivery. Am J Obstet Gynecol. 1995;172 1 pt 1:44-50.

12. Wilkins-Haug L, Quade B, Morton CC. Confined placental mosaicism as a risk factor among newborns with fetal growth restriction. Prenat Diagn. 2006;26:428-432.

13. Stipoljev F, Latin V, Kos M, Miskovic B, Kurjak A. Correlation of confined placental mosaicism with fetal intrauterine growth retardation. A case control study of placentas at delivery. Fetal Diagn Ther. 2001;16:4-9.

14. Groli C, Cerri V, Tarantini M, et al. Maternal serum screening and trisomy 16 confined to the placenta. Prenat Diagn. 1996;16:685-689.

15. Shaffer LG, Langlois S, McCaskill C, et al. Analysis of nine pregnancies with confined placental mosaicism for trisomy 2. Prenat Diagn. 1996;16:899-905.

16. Fryburg JS, Dimaio MS, Yang-Feng TL, Mahoney MJ. Follow-up of pregnancies complicated by placental mosaicism diagnosed by chorionic villus sampling. Prenat Diagn. 1993;13:481-494.

17. Wolstenholme J, Rooney DE, Davison EV. Confined placental mosaicism, IUGR, and adverse pregnancy outcome: a controlled retrospective U.K. collaborative survey. Prenat Diagn. 1994;14:345-361.

18. Schwinger $E$, Seidl $E$, Klink F, Rehder $H$. Chromosome mosaicism of the placenta-a cause of developmental failure of the fetus? Prenat Diagn. 1989:9:639-647.

19. Dorfmann AD, Perszyk J, Robinson P, Black SH, Schulman JD. Rare nonmosaic trisomies in chorionic villus tissue not confirmed at amniocentesis. Prenat Diagn. 1992;12:899-902.

20. Kennerknecht I, Krämer S, Grab D, Terinde R, Vogel W. A prospective cytogenetic study of third-trimester placentae in small-for-date but otherwise normal newborns. Prenat Diagn. 1993;13:257-269.

21. Amor DJ, Neo WT, Waters E, Heussler H, Pertile M, Halliday J. Health and developmental outcome of children following prenatal diagnosis of confined placental mosaicism. Prenat Diagn. 2006:26:443-448.

22. Baffero GM, Somigliana E, Crovetto $F$, et al. Confined placental mosaicism at chorionic villous sampling: risk factors and pregnancy outcome. Prenat Diagn. 2012;32:1102-1108.
23. Grati FR, Grimi B, Frascoli G, et al. Confirmation of mosaicism and uniparental disomy in amniocytes, after detection of mosaic chromosome abnormalities in chorionic villi. Eur J Hum Genet. 2006;14:282-288.

24. Grati FR, Bajaj K, Malvestiti F, Agrati C, et al. The type of feto-placental aneuploidy detected by cfDNA testing may influence the choice of confirmatory diagnostic procedure. Prenat Diagn. 2015;35: 994-998.

25. Villar J, Cheikh Ismail L, et al. International standards for newborn weight, length, and head circumference by gestational age and sex: the Newborn Cross-Sectional Study of the INTERGROWTH-21st Project. Lancet. 2014;384:857-868.

26. Hadlock FP, Harrist RB, Martinez-Poyer J. In utero analysis of fetal growth: a sonographic weight standard. Radiology. 1991;181:129-133.

27. Yudkin PL, Aboualfa M, Eyre JA, Redman CW, Wilkinson AR. New birthweight and head circumference centiles for gestational ages 24 to 42 weeks. Early Hum Dev. 1987; 15:45-52.

28. Benn P. Trisomy 16 and trisomy 16 mosaicism: a review. Am J Med Genet. 1998;79:121-133.

29. Yong PJ, Barrett IJ, Kalousek DK, Robinson WP. Clinical aspects, prenatal diagnosis, and pathogenesis of trisomy 16 mosaicism. J Med Genet. 2003:40:175-182.

30. Tørring N, Petersen OB, Becher N, Vogel I, Uldbjerg N, Danish Fetal Medicine Study Group; Danish Clinical Genetics Study Group. First trimester screening for other trisomies than trisomy 21, 18, and 13. Prenat Diagn. 2015;35:612-619.

31. Grau Madsen S, Uldbjerg N, Sunde L, Becher N, Danish Fetal Medicine Study Group, Danish Clinical Genetics Study Group. Prognosis for pregnancies with trisomy 16 confined to the placenta: a Danish cohort study. Prenat Diagn. 2018;38:1103-1110.

32. Miura K, Yoshiura K, Miura S, et al. Clinical outcome of infants with confined placental mosaicism and intrauterine growth restriction of unknown cause. Am J Med Genet A. 2006;140A:1827-1833.

33. Benn P, Grati FR. Genome-wide non-invasive prenatal screening for all cytogenetically visible imbalances. Ultrasound Obstet Gynecol. 2018;51:429-433.

34. Pertile MD, Halks-Miller M, Flowers N, et al. Rare autosomal trisomies, revealed by maternal plasma DNA sequencing, suggest increased risk of feto-placental disease. Sci Transl Med. 2017;9:eaan1240.

35. Fiorentino F, Bono S, Pizzuti F, et al. The clinical utility of genome-wide non invasive prenatal screening. Prenat Diagn. 2017;37:593-601.

36. Bianchi DW, Chiu RWK. Sequencing of circulating cell-free DNA during pregnancy. N Engl J Med. 2018;379:464-473.

37. Van Opstal D, van Maarle MC, Lichtenbelt K, et al. Origin and clinical relevance of chromosomal aberrations other than the common trisomies detected by genome-wide NIPS: results of the TRIDENT study. Genet Med. 2018;20:480-485.

38. Chatron N, Till M, Abel C, Bardel C, Ramond F, Sanlaville D, et al. Rare autosomal trisomies (RATs) detection through noninvasive prenatal testing (NIPT): benefits for pregnancy management. Ultrasound Obstet Gynecol. 2019;53:129-130.

39. Gregg AR, Skotko BG, Benkendorf JL. et al. Noninvasive prenatal screening for fetal aneuploidy, 2016 update: a position statement of the American College of Medical Genetics and Genomics. Genet Med. 2016;18:1056-1065. 